\title{
Monte Carlo Solution of High Electric Field Hole Transport Processes in Avalanche Amorphous Selenium
}

\author{
Atreyo Mukherjee, ${ }^{\dagger}$ Dragica Vasileska, ${ }^{\ddagger}$ John Akis, ${ }^{\mathbb{I}}$ and Amir H. Goldan*, II \\ †Department of Electrical Engineering, College of Engineering and Applied Sciences, Stony Brook \\ University, Stony Brook, NY, US \\ $\ddagger$ School of Electrical, Computer and Energy Engineering, Arizona State University, Tempe, AZ \\ IIDepartment of Radiology, School of Medicine, Stony Brook University, Stony Brook, NY \\ E-mail: Amirhossein.Goldan@stonybrookmedicine.edu
}

22 Pages, 7 Sections and 6 Figures 


\section{Supplementary S1. Multi-scale Simulation Pipeline}

At high electric fields, amorphous selenium ( $a$-Se) has the unique property of single carrier (hole) impact ionization, when the hole transport regime shifts from activated and localized hopping conduction to band-like non-activated extended states transport. In this work we shall, thus, restrict out attention to the avalanche regime of transport i.e., model the high electric field hole transport process in $a$-Se. The Ioffe-Regel criterion states that, a continuum of electrical and semiconducting properties can be observed across allotropic forms of a material that have a similarity in short to medium-range order. ${ }^{1+-3}$ Our simulation pipeline starts with molecular dynamics simulations, where we show strong correlations in simulated radial distribution functions (RDFs) up to $10 \AA$ between $a$-Se, and trigonal selenium (t-Se). ${ }^{4}$ Thus we showed that for various phase modifications in elemental selenium, similar electronic and vibrational properties arise from the retainment of the

local order (i.e., bond length, bond angle, and coordination number). ${ }^{5}$ Previously, such a similarity was also observed between alpha-quartz and amorphous silicon dioxide $\left(\mathrm{SiO}_{2}\right)$ (similar band-gap, band-structure, phonon spectra), which allowed Fischetti et al. to study hot electron transport in glassy $\mathrm{SiO}_{2}$ by treating the oxide as a perfect crystal. ${ }^{[67} \mathrm{Next}$, we used density functional theory (DFT) to calculate the electronic and phonon band structure and the valence band density of states (VB-DOS) in the crystalline counterpart of $a$-Se, that is $t$-Se as shown in Fig. S1. The parameters calculated using DFT are coupled to an in-house Monte Carlo (MC) simulation framework that solves the semiclassical Boltzmann transport equation (BTE), ${ }^{8}$ to gain insight into the extended state high field hole-transport process in $a$-Se, by modeling the band-transport lattice theory of its crystalline counterpart $t$-Se. The lack of long-range order in the amorphous phase was modeled using three additional scattering processes, namely polar optical phonon scattering, hole-dipole scattering and disorder scattering. 

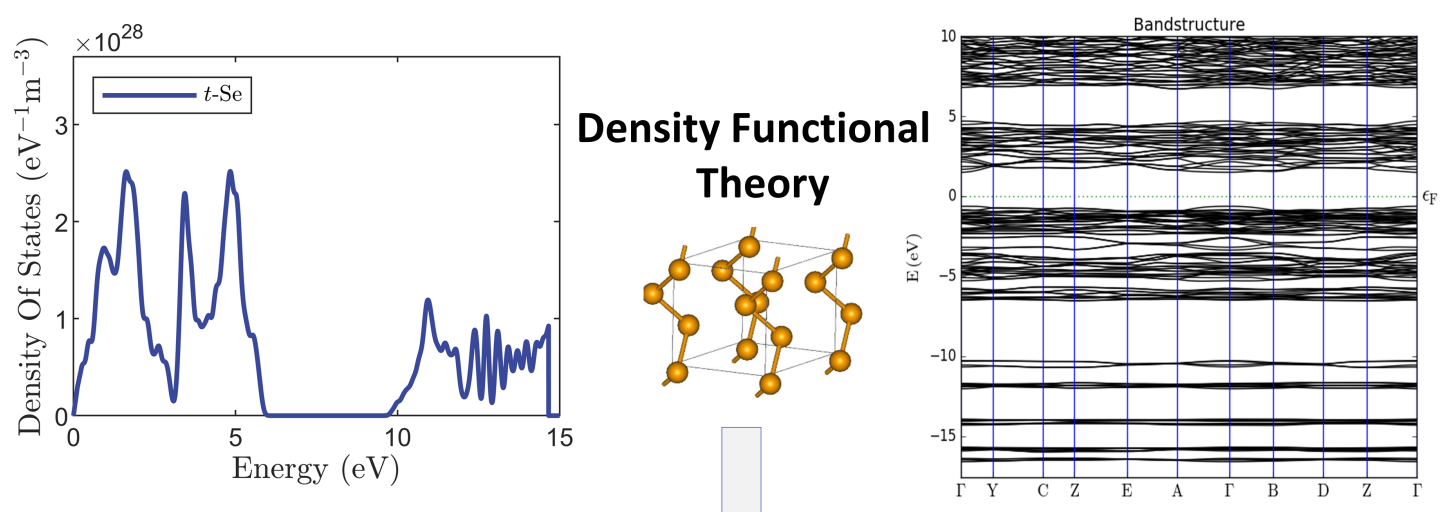

\section{Experimental Hole-Phonon Coupling Parameters}

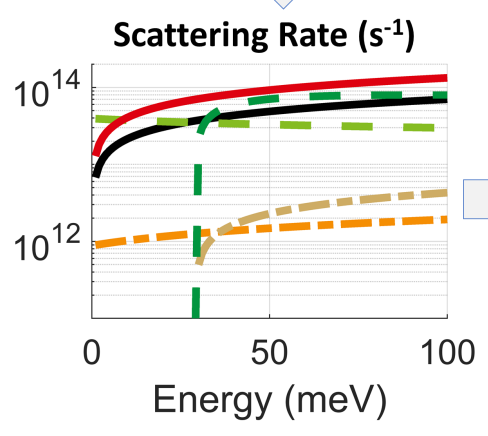

Ensemble Monte Carlo Monte Carlo

\section{Multiscale Modeling Approach}

Figure S1: Simulation pipeline showing DFT calculated VB-DOS and energy bandstructure, along with experimental hole-phonon coupling parameters, used in the scattering rate calculation in $a$-Se. The scattering rates thus calculated is used in the Monte Carlo simulation framework.

\section{Supplementary S2. Monte Carlo Solution to Boltzmann Trans- port Equation}

The electrical behavior of charge carriers in a material can be predicted by solving the BTE, which defines $f_{\mathbf{k}}(\mathbf{r})$ as the probability distribution function for occupancy of a state $\mathbf{k}$ at position $\mathbf{r}$ with holes. The change per time interval $\partial t$ in the distribution function $\partial f_{\mathbf{k}}(\mathbf{r})$, due to force fields and concentration gradients, that leads to the movement of particles in 
real and $\mathbf{k}$ space, is given by: ${ }^{9}$

$$
\frac{\partial f_{\mathbf{k}}(\mathbf{r})}{\partial t}=-\frac{q_{\mathrm{e}} E_{\mathrm{f}}}{\hbar} \cdot \nabla_{\mathbf{k}} f_{\mathbf{k}}(\mathbf{r})+\left.\frac{\partial f_{\mathbf{k}}(\mathbf{r})}{\partial t}\right|_{\text {coll }}-\frac{1}{\hbar} \nabla_{\mathbf{k}} E_{\mathbf{k}} \cdot \nabla_{\mathbf{r}} f_{\mathbf{k}}(\mathbf{r})+s(\mathbf{r}, \mathbf{p}, t),
$$

where $E_{\mathrm{f}}$ refers to the applied uniform electric field and $q_{\mathrm{e}}$ is the electronic charge. The first term on the right hand side describes the drift processes as a result of the application of $E_{\mathrm{f}}$. The second term denotes the collision integral, which is the difference between in and out-of-state scattering processes. The third term denotes the diffusion processes which may occur due to temperature or concentration gradients. The fourth term denotes the generation and recombination processes and is approximated to be zero. In this work, we model drift phenomenon in a bulk material and are only concerned with the spatial spreading of carriers that occurs due to the application of $E_{\mathrm{f}}$. The MC-BTE with a pico-second (ps) time resolution is used to study the band-like (non-activated extended state) transport in selenium where the carrier drift time is much less than the trapping time. ${ }^{410}$ In steady state, the time derivative goes to zero, and the net rate of energy loss due to the different scattering mechanisms is balanced by the rate of energy gain from $E_{\mathrm{f}}$. In the long time limit, the MC technique ${ }^{11+19}$ gives a solution to the BTE (Eq. 1) from which we can obtain microscopic access to carrier trajectories and relaxation dynamics of non-equilibrium 'hot' holes in extended states, driven by scattering. The bulk MC theory considers an ensemble of particles which are accelerated in the direction of $E_{\mathrm{f}}$ (free flight) and their trajectory is interrupted by instantaneous scattering events. The scattering interactions decide the final energy and momentum of the particles. At a specific $E_{\mathrm{f}}$ and at the end of a sufficiently long simulation time (long enough for the energy to settle), ensemble parameters like velocity and energy are calculated. By modeling an ensemble of hole carriers representative of the physical system of interest (i.e. $a$-Se), the evolution of non stationary time-dependent carrier distributions under the influence of a time-dependent driving force may be simulated. The Generic flowchart of the Monte Carlo algorithm divided grossly into 4 tasks is shown in Fig. S2 10 


\section{Task 1: Parameters Initialization}

First, all simulation parameters (e.g. material and transport constants, number of particle attributes, simulation time increments, number of scattering mechanisms, maximum number of particles etc.) are defined in the parameters initialization subroutine as user inputs.

\section{Task 2: Scattering Table Construction}

Next, we proceed to the creation of the scattering table. The individual scattering rates for acoustic, polar and non-polar optical phonons, impact ionization, disorder and hole-dipole scattering, all as a function of energy go into the simulation. Subsequently, we calculate the cumulative rate $\Gamma_{\text {total }}(E)=\sum_{i=1}^{n} \Gamma_{\mathrm{i}}(E)+\Gamma_{\text {self-scatter }}(E)$, where $n$ denotes the total number of scattering mechanisms and $\Gamma_{\text {self-scatter }}(E)$ is the self-scattering term that does not change the energy or momentum of the carriers and merely adjusts itself in time so that $\Gamma_{\text {total }}(E)$ remains constant. ${ }^{20 \mid}$ We normalize the scattering table so that the selection of the scattering mechanism is determined by drawing a random number uniformly distributed between 0 and 1.

\section{Task 3: Carrier Initialization}

We initialize carriers' velocity, energy, and wavevectors. Our model tracks a total number of 1,000 carriers, to achieve a good compromise between accuracy and computational cost, and their energies and wave vectors are initialized according to the Maxwell-Boltzmann distribution. The initial energies of the carriers is set to $-\frac{3}{2} k_{\mathrm{B}} T * \log \left(r_{\mathrm{n}}\right)$, where $r_{\mathrm{n}}$ represents a pseudo-random number generated between $0-1$ and $\mathbf{k}$ is calculated from the non-parabolic $E-\mathbf{k}$ relation. 


\section{Task 4: Free-Flight and Scatter time loop.}

To obtain the time evolution of carriers, the simulation is divided into time-steps of $\Delta t$ during which the free-flight subroutine is called and at the end of which the ensemble averages are calculated. At first every carrier drifts freely as per the applied electric field. The free-flight time is calculated as $t_{\mathrm{i}}=-\log \left(r_{\mathrm{n}}\right) / \Gamma_{\max }$, where $\Gamma_{\max }$ refers to the maximum scattering rate. After a carrier undergoes free-flight, and given that changes in momentum result in changes in energy, a suitable scattering mechanism is selected based on this new energy and a random number from the cumulative normalized scattering table. The final state of the particle is then calculated, depending on the type of the scattering event, and changes in angle and energy are accounted for at the end of the drift process. The angle after scattering depends on the scattering mechanism being isotropic or an-isotropic . Isotropic scattering mechanism favors scattering in all directions equally and thus the direction of the wavevector is randomized. The magnitude of the wavevector $\mathbf{k}$ is then calculated from the new energy value using the non-parabolic $E-\mathbf{k}$ relationship.

According to the Ergodic theorem, time and spatial averages show similar behavior in a statistical dynamical system. This allowed the calculation of the TOF mobility, impact ionization gain and spatially averaged ensemble hole energy, as the trajectories of individual carriers are tracked and carriers were taken out of the simulation at the end of travel lengths, sufficiently long for steady state to be achieved. For spatial averaging, TOF and avalanche gain calculations, the simulation is continued until all carriers were collected as shown in Fig. S2. The TOF mobility is calculated as $\mu=L /\left(E * t_{\text {tr }}\right)$, where $L$ is the the length of the device, $E$ is the applied electric field and $t_{\text {tr }}$ is the transit time of holes to cross the length of the device. The impact ionization gain is calculated by dividing the number of carriers at the end of travel by the initial number of carriers. 


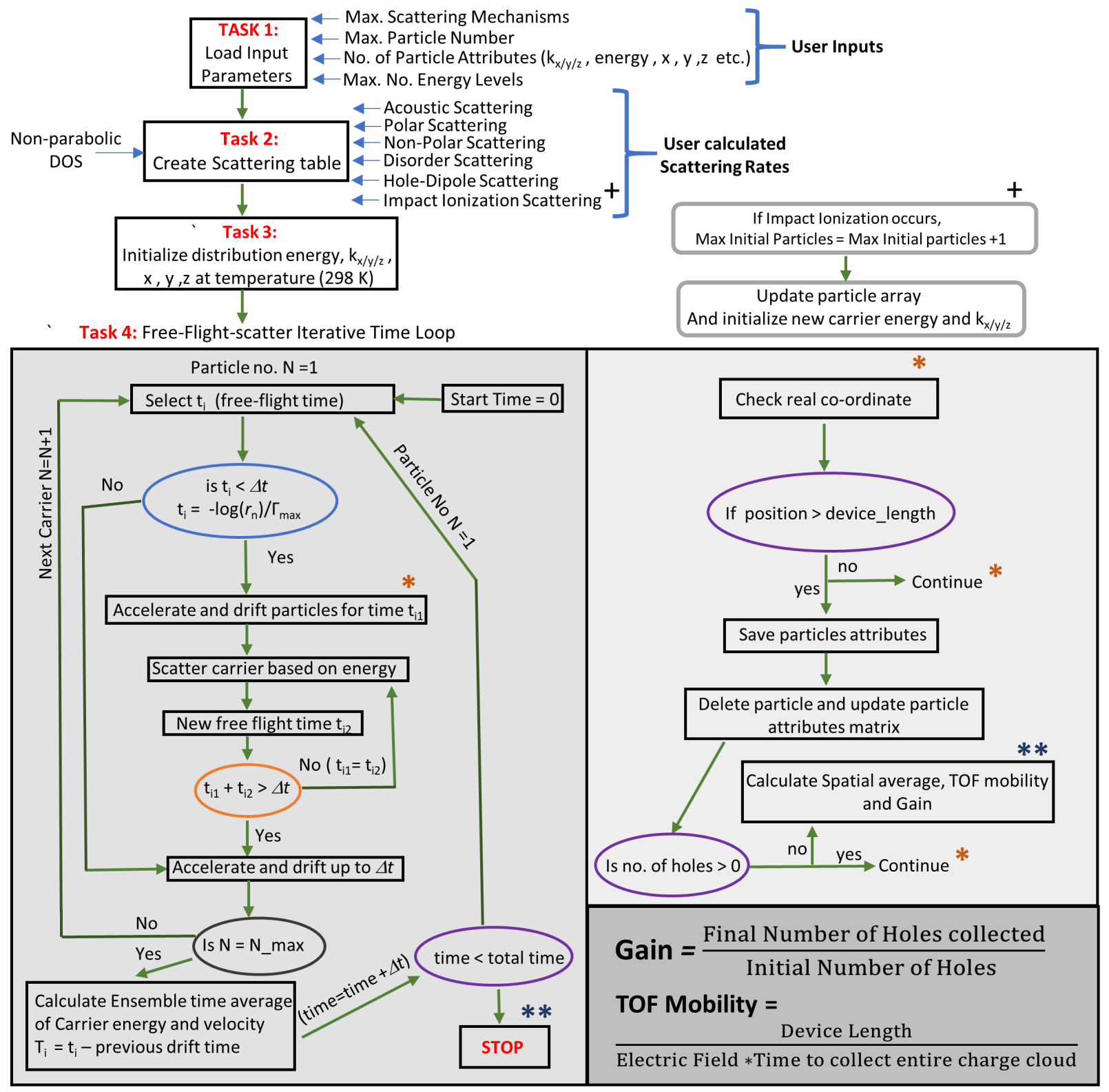

Figure S2: Flow-chart for a generic bulk Monte Carlo algorithm containing all relevant subroutines. 


\section{Supplementary S3. Scattering Rate Derivations}

The transition probability from initial state $|\mathbf{k}\rangle$ to final state $\left|\mathbf{k}^{\prime}\right\rangle$ is calculated using Fermi's golden rule. ${ }^{2122} \mathrm{~A}$ summation over all final states in converted into an integral, to calculate the total scattering rate out of state $|\mathbf{k}\rangle$ :

$$
\frac{1}{\tau(\mathbf{k})}=\frac{2 \pi}{\hbar} \frac{V}{(2 \pi)^{3}} \int_{0}^{2 \pi} \mathrm{d} \phi \int_{0}^{\pi} \sin \theta \mathrm{d} \theta \int_{0}^{\infty}\left|M\left(\mathbf{k}, \mathbf{k}^{\prime}\right)\right|^{2} \delta\left(E_{\mathbf{k}^{\prime}}-E_{\mathbf{k}} \mp \hbar \omega_{\mathbf{q}}\right) \mathbf{k}^{\prime 2} \mathrm{~d} \mathbf{k}^{\prime},
$$

where $V$ is the volume, and $\left|M\left(\mathbf{k}, \mathbf{k}^{\prime}\right)\right|$ is the matrix element, which is unique to every scattering mechanism. When $\left|M\left(\mathbf{k}, \mathbf{k}^{\prime}\right)\right|$ is independent of the phonon wavevector, it can be taken out of the integral and the final expression is shown in Eq. 3. This solution holds true for different scattering mechanisms as long as $\left|M\left(\mathbf{k}, \mathbf{k}^{\prime}\right)\right|$ remains constant. This further implies that the scattering process is isotropic. Hence, the total scattering rate out of state $\mathbf{k}$ is given by:

$$
\frac{1}{\tau(\mathbf{k})}=\frac{2 \pi V}{\hbar}\left|M\left(\mathbf{k}, \mathbf{k}^{\prime}\right)\right|^{2} \frac{1}{2} g_{c}\left(E_{\mathbf{k}} \pm \hbar \omega_{\mathbf{q}}\right) .
$$

In Eq. 3, $g_{c}\left(E_{\mathbf{k}^{\prime}}\right)=\left(\sqrt{2} m_{d^{\frac{3}{2}}} / \pi^{2} \hbar^{3}\right) \sqrt{E_{\mathbf{k}^{\prime}}\left(1+\alpha E_{\mathbf{k}^{\prime}}\right)}\left(1+2 \alpha E_{\mathbf{k}^{\prime}}\right)$ is the 3D non-parabolic DOS.

Acoustic Phonons Scattering : Under the elastic and equipartition approximation, the expression for $\left|M\left(\mathbf{k}, \mathbf{k}^{\prime}\right)\right|^{2}$ for acoustic phonon scattering is:112

$$
\left|M\left(\mathbf{k}, \mathbf{k}^{\prime}\right)\right|^{2}=\frac{\Xi_{\mathrm{ac}}^{2} k_{\mathrm{B}} T}{2 \rho V v_{\mathrm{s}}^{2}} .
$$

where $\rho$ is the crystal density, $\Xi_{a c}$ is the average acoustic deformation potential and $v_{\mathrm{S}}$ is the average sound velocity. Inserting Eq. 4 in Eq. 3 we obtain the total acoustic phonon 
scattering rate:

$$
\frac{1}{\tau(\mathbf{k})}=\frac{2 \pi}{\hbar} \frac{\Xi_{\mathrm{ac}}^{2} k_{\mathrm{B}} T}{2 \rho v_{\mathrm{s}}^{2}} \frac{1}{2} g_{\mathrm{c}}\left(E_{\mathbf{k}^{\prime}}\right)
$$

Higher values of the deformation potential constant and lower values of the sound velocities lead to higher scattering rates and lower mobilities of holes (closer to that of $a$-Se where $\mu \approx 1.5 \mathrm{~cm}^{2} \mathrm{~V}^{-1} \mathrm{~s}^{-1}$ ). Here, we have made the assumption that the carriers (holes) in $t$-Se traveling in the perpendicular direction to the $c$-axis, propagate in $a$-Se. The interaction of holes with acoustic phonons is then treated in $a$-Se in an average sense with average sound velocities and deformation potentials. The average acoustic scattering rate for $a$-Se is calculated as follows: $1 / \tau(\mathbf{k})_{a-S e}=1 / \tau(\mathbf{k})_{t-S e_{\perp}}$ ( $t$ refers to trigonal), thus leading to Eq. 6

$$
\frac{1}{\tau(\mathbf{k})_{a-S e}}=\frac{2 \pi}{\hbar} \frac{\left(\Xi_{\mathrm{ac} \perp}^{2}\right) k_{\mathrm{B}} T}{2 \rho v_{\mathrm{s} \perp}^{2}} \frac{1}{2} g_{\mathrm{c}}\left(E_{\mathbf{k}^{\prime}}\right)
$$

Non-Polar Optical Phonon Scattering : The isotropic and $q$-independent matrix element $\left|M\left(\mathbf{k}, \mathbf{k}^{\prime}\right)\right|^{2}$ is of the form 12

$$
\left|M\left(\mathbf{k}, \mathbf{k}^{\prime}\right)\right|^{2}=\frac{\hbar D_{\mathrm{o}}^{2}}{2 \rho V \omega_{\mathrm{o}}}\left(n\left(\omega_{\mathrm{o}}\right)+\frac{1}{2} \mp \frac{1}{2}\right)
$$

where $D_{\mathrm{o}}$ refers to the optical (or zeroth order) deformation potential once again calculated from the non-polar optical scattering rates in the $\perp$ direction to the $c$-axis in $t$-Se. Since the matrix element is q-independent we can substitute Eq.71into Eq. 3 to obtain,

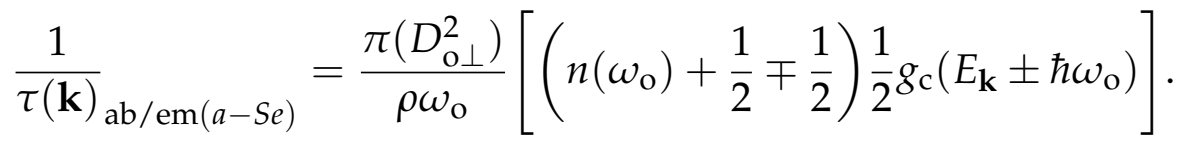

Adding Disorder : Modeling transport of holes requires us to account for the lack 
of long range order in the amorphous phase in selenium. We assume that the principal effect of the disorder is to produce elastic hole-lattice scattering whose scattering rate is governed by the final density of states (as shown in Eq. 3) , i.e. $\left|M\left(\mathbf{k}, \mathbf{k}^{\prime}\right)\right|^{2}$ is an adjustable constant in the simulation. ${ }^{23}$ This corresponds to a short-range order parameter $\approx 10 \AA$ as seen from the reduced radial distribution function (RDF) of $a$-Se obtained using Molecular Dynamics simulations and comparing it with that of $t$-Se. $\frac{415}{45}$

Hole-Dipole Scattering: In practice, a distribution of dipole moments is expected depending on the separation of atoms and the charges they bear. If the electric dipole moment is $\mathbf{M}$, then the scattering potential due to a screened dipole is of the form:2124

$$
V(\mathbf{r})=\frac{q_{e} \mathbf{M r}}{4 \pi \epsilon r^{3}}\left(1+\frac{r}{L_{\mathrm{D}}}\right) e^{-r / L_{\mathrm{D}}},
$$

where $q_{\mathrm{e}}$ is the electronic charge, $L_{\mathrm{D}}$ is the Debye screening length and $\mathrm{r}$ is the real space coordinate of the hole. The dot product in Eq. 9 can be expanded as

$$
V(\mathbf{r})=\frac{q_{e}|\mathbf{M}|}{4 \pi \epsilon r^{2}} \cos (\mathbf{M}, \mathbf{r})\left(1+\frac{r}{L_{\mathrm{D}}}\right) e^{-r / L_{\mathrm{D}}}
$$

where the dipole moment $\mathbf{M}$ and the real space co-ordinate of holes $\mathbf{r}$ have polar angles $(u, v)$, and $(\theta, \phi)$, with respect to $\beta$ as polar axis, where $\beta=\mathbf{k}^{\prime}-\mathbf{k}$. This yields,

$$
V(\mathbf{r})=\frac{q_{e}|\mathbf{M}|}{4 \pi \epsilon r^{2}}[\cos (u) \cos (\theta)+\sin (u) \sin (\theta) \cos (\phi-v)]\left(1+\frac{r}{L_{\mathrm{D}}}\right) e^{-r / L_{\mathrm{D}}} .
$$

The matrix element $M\left(\mathbf{k}, \mathbf{k}^{\prime}\right)$ is evaluated from

$$
M\left(\mathbf{k}, \mathbf{k}^{\prime}\right)=\frac{1}{V} \cdot \iiint \mathrm{d}^{3} r V(\mathbf{r}) e^{i \beta . \mathbf{r}},
$$

where $V$ represents the normalized volume. We have chosen that $\beta$ is aligned with the 
polar axis. Hence :

$$
\beta . \mathbf{r}=\beta r \cos (\theta)
$$

With this choice of a co-ordinate system and substituting Eq.13 in Eq. 12, we have

$$
M\left(\mathbf{k}, \mathbf{k}^{\prime}\right)=\frac{1}{V} \int_{0}^{\infty} r^{2} \mathrm{~d} r \int_{-1}^{1} \mathrm{~d}(\cos (\theta)) \int_{0}^{2 \pi} \mathrm{d} \phi V(\mathbf{r}) e^{i \beta r \cos (\theta)},
$$

which further leads to:

$$
\begin{aligned}
M\left(\mathbf{k}, \mathbf{k}^{\prime}\right)=\frac{q_{e}|\mathbf{M}|}{4 \pi \epsilon V} \int_{0}^{\infty}\left(1+\frac{r}{L_{\mathrm{D}}}\right) e^{-r / L_{\mathrm{D}}} \mathrm{d} r \int_{-1}^{1} e^{i \beta r \cos (\theta)} \mathrm{d}(\cos (\theta)) \\
\quad \int_{0}^{2 \pi}[\cos (u) \cos (\theta)+\sin (u) \sin (\theta) \cos (\phi-v)] \mathrm{d} \phi .
\end{aligned}
$$

While carrying over the integration over $\phi$, the contribution from the $\sin (u) \sin (\theta) \cos (\phi-$ $v)$ term disappears. The first term gives a factor of $2 \pi$. Thus, we get:

$$
M\left(\mathbf{k}, \mathbf{k}^{\prime}\right)=\frac{q_{e}|\mathbf{M}| \cos (u)}{2 \epsilon V} \int_{0}^{\infty}\left(1+\frac{r}{L_{\mathrm{D}}}\right) e^{-r / L_{\mathrm{D}}} \mathrm{d} r \int_{-1}^{1} e^{i \beta r \cos (\theta)} \cos (\theta) \mathrm{d}(\cos (\theta)) .
$$

Next, we solve the integration under $\theta$ by parts to obtain,

$$
M\left(\mathbf{k}, \mathbf{k}^{\prime}\right)=\frac{q_{e}|\mathbf{M}| \cos (u)}{i \epsilon V} \int_{0}^{\infty}\left(\frac{\cos (\beta r)}{\beta r}-\frac{\sin (\beta r)}{(\beta r)^{2}}\right)\left(1+\frac{r}{L_{\mathrm{D}}}\right) e^{-r / L_{\mathrm{D}}} \mathrm{d} r,
$$

where $i$ is the imaginary number. An average is next taken over all possible orientations of the dipoles, i.e. $\cos (u)$ is replaced by its average value of $l / \sqrt{3}$, which, in turn, results in:

$$
M\left(\mathbf{k}, \mathbf{k}^{\prime}\right)=\frac{q_{e}|\mathbf{M}|}{i \sqrt{3} \epsilon V} \int_{0}^{\infty}\left(\frac{\cos (\beta r)}{\beta r}-\frac{\sin (\beta r)}{(\beta r)^{2}}\right)\left(1+\frac{r}{L_{\mathrm{D}}}\right) e^{-r / L_{\mathrm{D}}} \mathrm{d} r .
$$


Finally, we evaluate this integral to obtain the following form of the matrix element,

$$
M\left(\mathbf{k}, \mathbf{k}^{\prime}\right)=\frac{q_{e}|\mathbf{M}| L_{\mathrm{D}}}{i \sqrt{3} \epsilon V}\left(\frac{\beta L_{\mathrm{D}}}{1+\beta^{2} L_{\mathrm{D}}^{2}}\right) .
$$

The total scattering probability out of state $\mathrm{k}$ is:

$$
\begin{aligned}
P(\mathbf{k})=\frac{V}{8 \pi^{3}} \frac{2 \pi T}{\hbar} & \int_{0}^{\infty} \mathbf{k}^{\prime 2} \mathrm{~d} \mathbf{k}^{\prime} \int_{-1}^{1} \mathrm{~d}(\cos (\theta)) \int_{0}^{2 \pi} \mathrm{d} \phi\left|M\left(\mathbf{k}, \mathbf{k}^{\prime}\right)\right|^{2} \delta\left(E_{\mathbf{k}^{\prime}}-E_{\mathbf{k}}\right) \\
& =\int_{-1}^{1} \mathrm{~d}(\cos (\theta)) \int_{0}^{2 \pi} \mathrm{d} \phi \underbrace{\frac{V T}{4 \pi^{2} \hbar} \int_{0}^{\infty} \mathbf{k}^{\prime 2} \mathrm{~d} \mathbf{k}^{\prime}\left|M\left(\mathbf{k}, \mathbf{k}^{\prime}\right)\right|^{2} \delta\left(E_{\mathbf{k}^{\prime}}-E_{\mathbf{k}}\right)}_{P(\theta, \phi)} .
\end{aligned}
$$

Since we assume non-parabolic bands, we have

$$
E_{\mathbf{k}^{\prime}}\left(1+\alpha E_{\mathbf{k}^{\prime}}\right)=\hbar^{2} \mathbf{k}^{\prime 2} / 2 m, \quad \mathrm{~d} \mathbf{k}^{\prime}=\frac{\mathrm{d} E_{\mathbf{k}^{\prime}}\left(1+2 \alpha E_{\mathbf{k}^{\prime}}\right) m}{\hbar^{2} \mathbf{k}^{\prime}},
$$

where $m$ is the effective mass and $\alpha$ is the non-parabolicity factor. We can further simplify the expression for $P(\theta, \phi)$ as follows:

$$
P(\theta, \phi)=\frac{V T m \mathbf{k}^{\prime}}{4 \pi^{2} \hbar^{3}} \int_{0}^{\infty} \mathrm{d} E_{\mathbf{k}^{\prime}}\left(1+2 \alpha E_{\mathbf{k}^{\prime}}\right)\left|M\left(\mathbf{k}, \mathbf{k}^{\prime}\right)\right|^{2} \delta\left(E_{\mathbf{k}^{\prime}}-E_{\mathbf{k}}\right) .
$$

The $\delta$ function in the integral requires $E_{\mathbf{k}^{\prime}}=E_{\mathbf{k}}$, which means that the initial and the final wave-vectors have the same magnitude $\left(\left|k^{\prime}\right|=|k|\right)$. This, in turns, means that we can immediately perform this integration to get

$$
P(\theta, \phi)=\frac{V T m k}{4 \pi^{2} \hbar^{3}}\left(1+2 \alpha E_{\mathbf{k}}\right)\left|M\left(\mathbf{k}, \mathbf{k}^{\prime}\right)\right|^{2} .
$$

Now, let $T$ be the time required for the hole to traverse a box of length $L_{z}$ with velocity 
v, i.e.,

$$
T=\frac{L_{\mathbf{z}}}{v}=\frac{L_{\mathrm{z}}}{\hbar k} m\left(\left(1+2 \alpha E_{\mathbf{k}}\right)=\frac{V}{A} \frac{m\left(\left(1+2 \alpha E_{\mathbf{k}}\right)\right.}{\hbar k},\right.
$$

where $A$ is the cross-section of the box and $V=L_{z} A$ is the volume of the box.

Substituting Eq. 24 in Eq. 23, we get:

$$
P(\theta, \phi)=\frac{V^{2} m}{4 \pi^{2} \hbar^{4}}\left(1+2 \alpha E_{\mathbf{k}}\right)^{2} \frac{\left|M\left(\mathbf{k}, \mathbf{k}^{\prime}\right)\right|^{2}}{A}=\frac{\sigma(\theta, \phi)}{A}
$$

where $\sigma(\theta, \phi)$ is the scattering cross-section. To get the total scattering cross-section $\sigma_{\mathrm{s}}$ we need to integrate over the entire solid angle, i.e.,

$$
\begin{aligned}
\sigma_{\mathrm{s}}=\int_{-1}^{1} \mathrm{~d}(\cos (\theta)) \int_{0}^{2 \pi} \mathrm{d} \phi & \sigma(\theta, \phi) \\
& =\frac{m^{2} q_{e}^{2} L_{\mathrm{D}}^{2}|\mathbf{M}|^{2}}{6 \pi \hbar^{4} \epsilon^{2}}\left(1+2 \alpha E_{\mathbf{k}}\right)^{2} \int_{-1}^{1}\left(\frac{\beta L_{\mathrm{D}}}{1+\beta^{2} L_{\mathrm{D}}^{2}}\right) \mathrm{d}(\cos (\theta)) .
\end{aligned}
$$

In Eq. $26|\mathbf{M}|=q_{e} a_{o}$, where $a_{o}$ represents the dipole radius which is of the order of the nearest neighbor distance and $\beta^{2}=4 k^{2} \sin ^{2}(\theta / 2)$. The integral is evaluated next to arrive at the following result,

$$
\sigma_{\mathrm{s}}=\frac{m^{2} q_{e}^{4} a_{o}^{2} L_{\mathrm{D}}^{2}}{3 \pi \hbar^{4} \epsilon^{2}}\left(1+2 \alpha E_{\mathbf{k}}\right)^{2} \frac{1}{\varrho^{2}}\left(\log \left(1+\varrho^{2}\right)+\frac{1}{1+\varrho^{2}}-1\right)
$$

where,

$$
\varrho^{2}=4 k^{2} L_{\mathrm{D}}^{2}
$$

Finally, the scattering rate for the hole-dipole interaction can is evaluated from the scattering cross as follows: 


$$
\begin{aligned}
& \frac{1}{\tau(\mathbf{k})}_{(a-S e)}=\sigma_{\mathrm{s}} N_{\mathrm{T}} v \\
& \quad=\frac{m q_{e}^{4} a_{o}^{2} L_{\mathrm{D}}^{2} N_{\mathrm{T}}}{3 \pi \hbar^{3} \epsilon^{2}} \frac{\sqrt{2 m E_{\mathbf{k}}\left(1+\alpha E_{\mathbf{k}}\right)}}{\hbar}\left(1+2 \alpha E_{\mathbf{k}}\right) \frac{1}{\varrho^{2}}\left(\log \left(1+\varrho^{2}\right)+\frac{1}{1+\varrho^{2}}-1\right),
\end{aligned}
$$

where $N_{\mathrm{T}}$ is the density of scattering dipole pairs.

Polar Optical Scattering : The difference in short and long-wavelength optical displacements of ions leads to macroscopic polarization of $a$-Se, which is evident from infrared reflectivity measurements that show fairly strong optical phonon coupling in the chalcogenide family. The vibrations of the crystal lattice lead to oscillations of this dipole, which acts as a scattering potential. The transition from state $|\mathbf{k}\rangle$ to $\left|\mathbf{k}^{\prime}\right\rangle$ is solved using the Random Phase Model for amorphous materials as described by Hindley 25126.

$$
\left|M\left(\mathbf{k}, \mathbf{k}^{\prime}\right)\right|^{2}=\frac{2 \pi q_{\mathrm{e}}^{2} \hbar \omega_{\mathrm{o}} a^{3}}{V^{2} q^{2}}\left(\frac{1}{\epsilon_{\infty}}-\frac{1}{\epsilon_{0}}\right)\left(n\left(\omega_{\mathrm{o}}\right)+\frac{1}{2}, \mp \frac{1}{2}\right)
$$

where $\epsilon_{\infty}$ and $\epsilon_{0}$ are the high and low frequency dielectric constants in $a-\mathrm{Se}, \mathrm{V}$ is the volume and $\mathrm{a}^{3}$ is the volume per atom. Since there are 3 atoms in the unit cell of $t$-Se: $\frac{a^{3}}{V}=$ $\frac{1}{3}$.

The final scattering rate is obtained by substituting Eq. 30 into Eq. 2 to get:

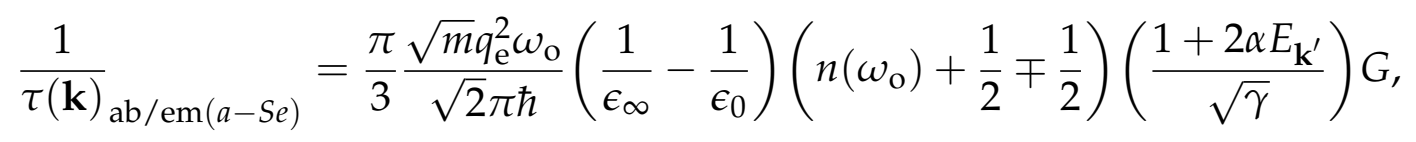

where,

$$
E_{\mathbf{k}^{\prime}}=E_{\mathbf{k}} \pm \hbar \omega_{\mathrm{o}}
$$




$$
\begin{gathered}
\gamma=E_{\mathbf{k}}\left(1+\alpha E_{\mathbf{k}}\right), \\
\gamma_{f}=E_{\mathbf{k}^{\prime}}\left(1+\alpha E_{\mathbf{k}^{\prime}}\right), \\
G=\frac{1}{2}\left(\log \frac{\gamma+\gamma_{f}+2 \sqrt{\gamma \gamma_{f}}}{\gamma+\gamma_{f}-2 \sqrt{\gamma \gamma_{f}}}\right) .
\end{gathered}
$$

Impact Ionization The impact ionization scattering mechanism in $a$-Se involves 2 holes and 1 electron, in which a 'hot' hole in the valence band ionizes an electron residing in the valence band across the bandgap into the conduction band, leading to an extra positive charge in the valence band and a negative charge in the conduction band. This scattering process is interpreted as the hole initiated impact ionization. The hole impact ionization scattering rate is next determined using an analytical result which was derived by Keldysh assuming a q-independent constant matrix element, and expanding the energies of the carriers involved in the impact ionization process with respect to the threshold energy $E_{\mathrm{t}}$ $\approx \mathrm{E}_{\mathrm{g}}(2.1 \mathrm{eV}) \cdot{ }^{27}$ Our impact ionization rate follows the single term Keldysh formula and was fitted to experimentally measured data. This led to the following expression for the total rate out of state $\mathbf{k}$ for impact ionization:

$$
\frac{1}{\tau(\mathbf{k})_{a-S e}}=8 X 10^{13}\left(E-E_{\mathrm{t}}\right)^{2}, 2.1 \mathrm{eV} \leq E \leq 5 \mathrm{eV}
$$

\section{Supplementary S4. Final State after Scattering}

In the ensemble Monte Carlo algorithm, a carrier free-flight (during which the particle dynamics is treated semi-classically according to Newtonian mechanics) is terminated with the scattering processes. A uniformly distributed random number between between 
0 and $\Gamma$, is used to select a scattering mechanism that terminated this free-flight (the final energy of the particle is used for this selection process):

$$
\Gamma=\Gamma_{\text {self }}[\mathbf{k}]+\Gamma_{1}[\mathbf{k}]+\Gamma_{2}[\mathbf{k}]+\Gamma_{3}[\mathbf{k}]+\ldots .+\Gamma_{N}[k],
$$

where $\mathbf{k}$ represents the wave-vector at the end of the free flight and $N$ is the number of scattering mechanisms.

After selecting the type of scattering mechanism, the final energy and momentum of the particle needs to be selected. For this specific selection $j$ where $1 \leq j \leq N$, the scattering rate $\Gamma_{j}\left[\mathbf{k} ; \mathbf{k}^{\prime}\right]$ is needed ( $\mathbf{k}$ and $\mathbf{k}^{\prime}$ are the particle wavevectors before and after scattering). For that purpose we define spherical coordinate system around the initial wavevector $\mathbf{k}$, whereas the final wavevector $\mathbf{k}^{\prime}$ is specified by $\left|\mathbf{k}^{\prime}\right|$ (which depends on the conservation of energy), as well as the polar and azimuthal angels $\theta$ and $\phi$ around $\mathbf{k}$. Typically, the scattering rate $\Gamma_{j}\left[\mathbf{k} ; \mathbf{k}^{\prime}\right]$ only depends on the angle $\theta$ between $\mathbf{k}$ and $\mathbf{k}^{\prime}$. Therefore, $\phi$ may be chosen using a uniform random number between 0 and $2 \pi$ (i.e., $2 \pi r$ ), while $\theta$ is chosen according to the cross-section for scattering arising from $\Gamma_{j}\left[\mathbf{k} ; \mathbf{k}^{\prime}\right] .12$

If the probability of scattering into a certain angle is integrable, an analytical formula may be derived to calculate the final angles after scattering. Otherwise, a rejection technique may be used to select the final angles.

Isotropic Scattering For scattering mechanisms where the matrix element, $\left|M\left(\mathbf{k}, \mathbf{k}^{\prime}\right)\right|$ is independent of the phonon wave-vector, $\theta$ can be readily calculated as $\cos (\theta)=1-2 r$ , where $r$ is random number uniformly distributed between 0 and 1. In our simulation, acoustic phonon scattering, non-polar optical phonon scattering, disorder scattering and impact ionization are treated as isotropic scattering processes.

Anisotropic Scattering : The anisotropic scattering processes are polar optical scattering and hole-dipole scattering. For polar optical phonon scattering an analytical formula 
can be derived for $\theta$, of the form:

$$
\cos (\theta)=\frac{(1+\zeta)-(1-\zeta)^{r}}{\zeta}
$$

where,

$$
\zeta=\frac{2 \sqrt{\gamma \gamma_{f}}}{\left(\sqrt{\gamma}-\sqrt{\gamma}_{f}\right)^{2}}
$$

$\gamma$ and $\gamma_{f}$ are calculated as shown in Eq 33 and Eq 34 , respectively, while $r$ is a randomly generated number uniformly distributed between 0 and 1 .

The direct technique described above can be applied when the integrals describing $\cos (\theta)$ in Supplementary S3., can be analytically calculated. For the hole-dipole scattering process, the integral cannot be easily solved. Thus, a rejection technique is employed as discussed by Jacoboni and Reggiani. $\cdot 15$

\section{Supplementary S5. Transient Characteristics}

The characteristic plots shown in this section are for qualitative validation of the code. We show that a simulation time of $50 \mathrm{ps}$ is sufficient to achieve steady state. In Fig. S3 the saturation of hole drift velocity is reached within a few picoseconds and this saturation value increases with increase in the electric field. Figure 54 shows the time evolution for average carrier energy. As expected, the steady state ensemble averaged energy increases with increase in electric field. 


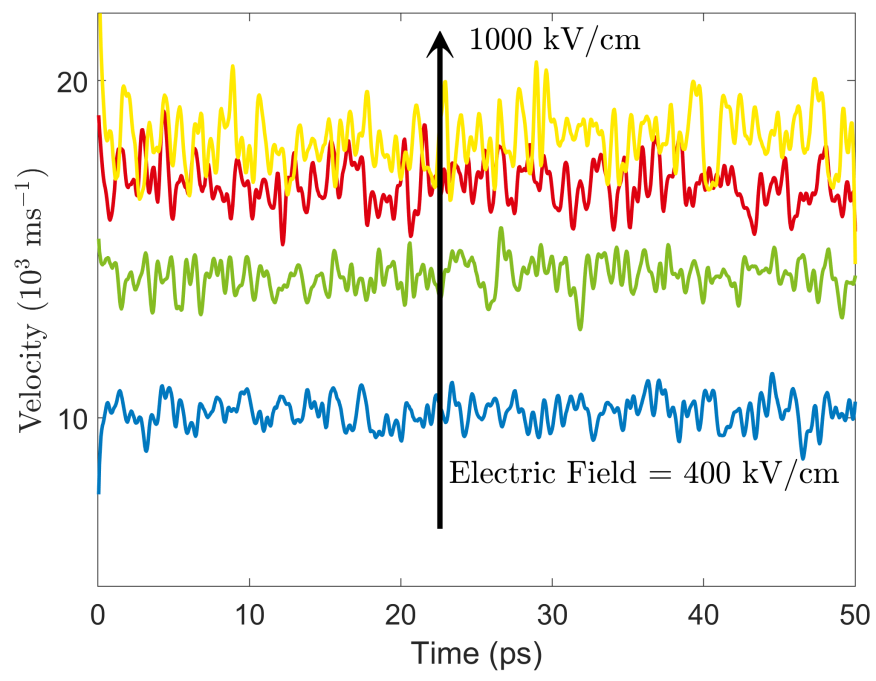

Figure S3: Time evolution of the hole drift velocity in $a$-Se. Electric field strengths range between 400 and $1000 \mathrm{kV} / \mathrm{cm}$, in $200 \mathrm{kV} / \mathrm{cm}$ increments.

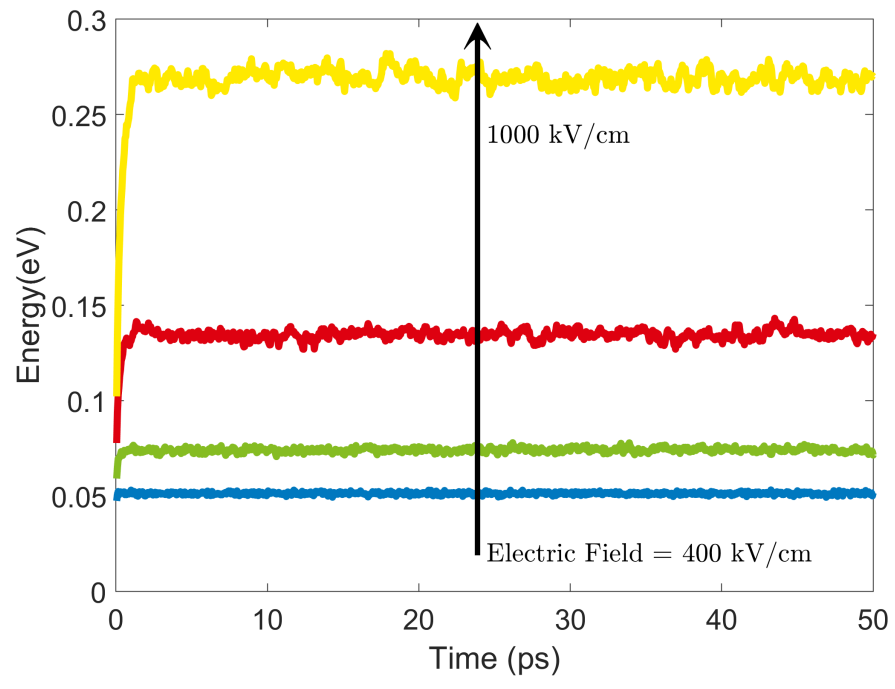

Figure S4: Time evolution of the average hole energy in in $a$-Se. Electric field strengths range between 400 and $1000 \mathrm{kV} / \mathrm{cm}$, in $200 \mathrm{kV} / \mathrm{cm}$ increments. 


\section{Supplementary S6. Impact Ionization Coefficient Calcula-}

\section{tion}

The impact ionization coefficient $\beta$, for holes, describes the number of hole-electron pairs generated by each hole per unit length of travel per unit time. $\beta$ is calculated using Eq. 40 for every simulation step,

$$
\beta=\frac{1}{n v_{\mathrm{n}}} \frac{d n}{d t} .
$$

In Eq. $40, d t$ is the simulation time step size which in our case is assumed to be $0.1 \mathrm{fs}$, $v_{\mathrm{n}}$ is the magnitude of the ensemble drift velocity at the end of every simulation step, $n$ is the number of carriers at the start of the simulation step and $d n$ is the total number of carriers generated during that time step. The simulation was stopped when steady state was reached. An ensemble average was taken between 0 and $75 \mathrm{ps}$ to calculate the MC-BTE impact ionization coefficients. A transient plot for the impact ionization coefficient at 1400 $\mathrm{kV} / \mathrm{cm}$ is shown in Fig. S5.

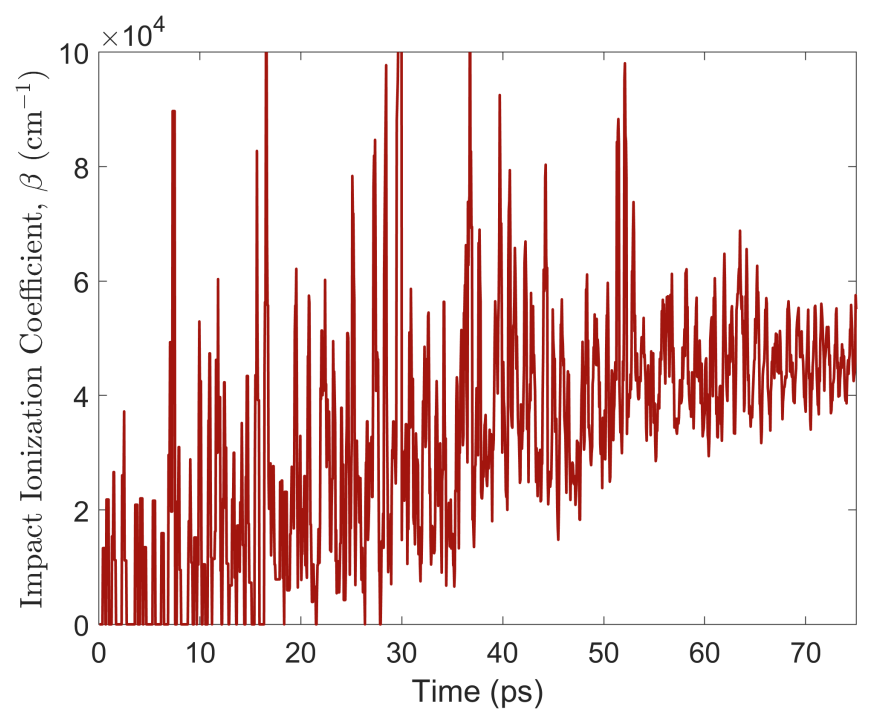

Figure S5: A transient plot showing the hole impact ionization coefficient calculated for electric field equal to $1400 \mathrm{kV} / \mathrm{cm}$. 


\section{Supplementary S7. Hole Energy distribution calculation}

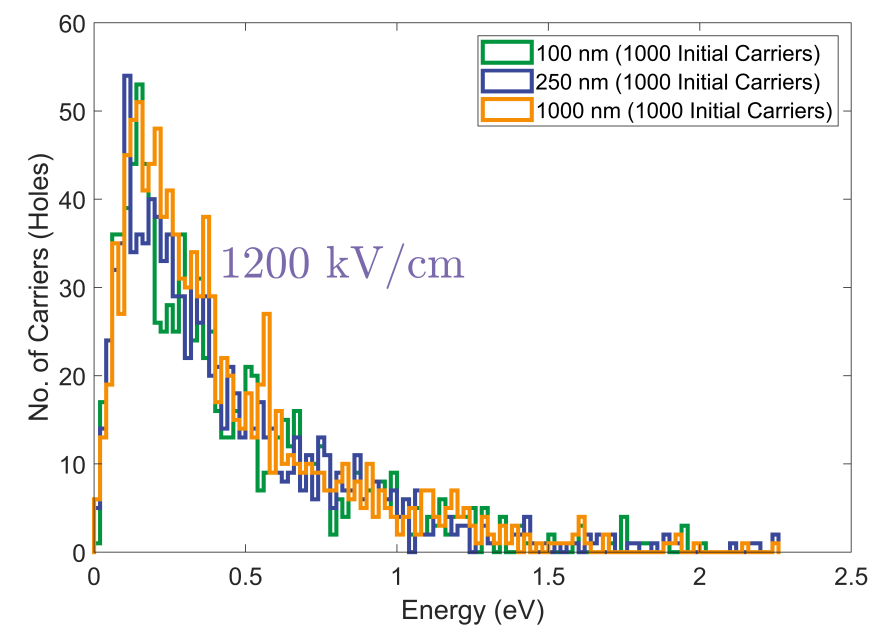

Figure S6: Histogram showing hole energy distribution at the end of travel for different drift lengths, at an electric field of $1200 \mathrm{kV} / \mathrm{cm}(100 \mathrm{~nm}$ Green, $250 \mathrm{~nm}$ Blue, and $1000 \mathrm{~nm}$ Orange). The distribution as well as ensemble spatial averages, saturate when we record the energies after a distance long enough for steady state $(50-100 \mathrm{~nm})$ to be obtained.

Fig. 56 shows the hole energy distribution obtained by following the histories of 1000 carriers and recording the energies at the end of travel over typical distances of $100 \mathrm{~nm}$, $250 \mathrm{~nm}$, and $1000 \mathrm{~nm}$. The spatially averaged energy distribution saturates once the energy values of individual carriers are recorded sufficiently far away from the initial position, for steady state to be achieved. In our simulation, the average steady state distance was calculated to be 50-100 nm. At higher electric fields the energy distributions saturated at shorter distances due to increased phonon scattering. 


\section{References}

(1) Ioffe, A. F.; Regel, A. R. Non-crystalline, amorphous, and liquid electronic semiconductors. Prog. Semicond. 1960, 4, 237-291.

(2) Skipetrov, S. E.; Sokolov, I. M. Ioffe-Regel criterion for Anderson localization in the model of resonant point scatterers. Phys. Rev. B 2018, 98.

(3) Gurvitch, M. Ioffe-Regel criterion and resistivity of metals. Phys. Rev. B 1981, 24.

(4) Mukherjee, A.; Vasileska, D.; Goldan, A. H. Hole transport in selenium semiconductors using density functional theory and bulk Monte Carlo. J. Appl. Phys 2018, 124, 235102.

(5) Goldan, A. H.; Li, C.; Pennycook, S. J.; Schneider, J.; Blom, A.; Zhao, W. Molecular structure of vapor-deposited amorphous selenium. J. Appl. Phys. 2016, 120, 135101.

(6) Ferry, D. K. The Physics and Technology of Amorphous $\mathrm{SiO}_{2}$; Plenum Press, 1988; pp 365-373.

(7) Fischetti, M. V.; DiMaria, D. J.; Brorson, S. D.; Theis, T. N.; Kirtley, J. R. Theory of high-field electron transport in silicon dioxide. Phys. Rev. B 1985, 31, 8124-8142.

(8) Fischetti, M. V.; Laux, S. E. Monte Carlo analysis of electron transport in small semiconductor devices including band-structure and space-charge effects. Phys. Rev. B 1988, 38, 9721.

(9) Péraud, J.-P. M.; Hadjiconstantinou, N. G. Efficient simulation of multidimensional phonon transport using energy-based variance-reduced Monte Carlo formulations. Phys. Rev. B 2011, 84.

(10) Mukherjee, A.; Akis, R.; Vasileska, D.; Goldan, A. A Monte Carlo solution to hole transport processes in avalanche selenium semiconductors. Physics and Simulation of Optoelectronic Devices XXVIII. 2020; p 112740U.

(11) Kalos, M. H.; Whitlock, P. A. Monte carlo methods; John Wiley \& Sons, 2009.

(12) Vasileska, D.; Goodnick, S. M.; Klimeck, G. Computational Electronics: Semiclassical and Quantum Device Modeling and Simulation; CRC press, 2017.

(13) Fawcett, W.; Boardman, A.; Swain, S. Monte Carlo determination of electron transport properties in gallium arsenide. J. Phys. Chem. Solids. 1970, 31, 1963-1990.

(14) Lebwohl, P. A. Monte Carlo simulation of response of a semiconductor to periodic perturbations. J. Appl. Phys. 1973, 44, 1744-1752. 
(15) Jacoboni, C.; Reggiani, L. The Monte Carlo method for the solution of charge transport in semiconductors with applications to covalent materials. Rev. Mod. Phys. 1983, 55, 645-705.

(16) Jacoboni, C.; Lugli, P. The Monte Carlo method for semiconductor device simulation; Springer Science \& Business Media, 2012.

(17) Barker, J. R.; Ferry, D. K. Self-Scattering Path-Variable Formulation of High-Field, TimeDependent, Quantum Kinetic Equations for Semiconductor Transport in the Finite-CollisionDuration Regime. Phys. Rev. Lett. 1979, 42, 1779-1781.

(18) Price, P. J. In Lasers, Junctions, Transport; Willardson, R., Beer, A. C., Eds.; Semiconductors and Semimetals; Elsevier, 1979; Vol. 14; pp 249 - 308.

(19) Hess, K. Monte Carlo device simulation: full band and beyond; Springer Science \& Business Media, 2012; Vol. 144.

(20) Rees, H. Calculation of distribution functions by exploiting the stability of the steady state. J. Phys. Chem. Solids 1969, 30, 643-655.

(21) Ridley, B. K. Quantum Processes in Semiconductors, 4th ed.; Oxford Science Publications, pp 1689-1699.

(22) Lundstrom, M. Fundamentals of Carrier Transport, 2nd edn; Cambridge University Press, 2000; $\mathrm{p}$ 434.

(23) Brust, D. Electronic Spectrum, $k$ Conservation, and Photoemission in Amorphous Germanium. Phys. Rev. Lett. 1969, 23, 1232-1234.

(24) Stratton, R. Dipole scattering from ion pairs in compensated semiconductors. J. Phys. Chem. Solids. 1962, 23, 1011-1017.

(25) Hindley, N. Random phase model of amorphous semiconductors II. Hot electrons. J. Non-Cryst. Solids 1970, 5, 31-40.

(26) Giner, C. T.; Comas, F. Electron-LO-phonon interaction in semiconductor double heterostructures. Phys. Rev. B 1988, 37, 4583.

(27) Tanaka, S. A lucky drift model, including a soft threshold energy, for the relation between gate and substrate currents in MOSFETs. Solid State Electron. 1989, 32, 935 - 946. 